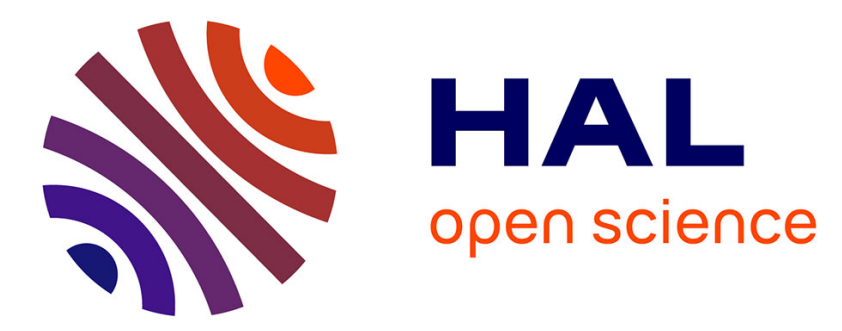

\title{
Photothermal microscopy: optical detection of small absorbers in scattering environments
}

\author{
Pierre Vermeulen, Laurent Cognet, Brahim Lounis
}

\section{To cite this version:}

Pierre Vermeulen, Laurent Cognet, Brahim Lounis. Photothermal microscopy: optical detection of small absorbers in scattering environments. Journal of Microscopy, 2014, pp.en ligne. 10.1111/jmi.12130 . hal-00998099

\section{HAL Id: hal-00998099 \\ https://hal.science/hal-00998099}

Submitted on 30 May 2014

HAL is a multi-disciplinary open access archive for the deposit and dissemination of scientific research documents, whether they are published or not. The documents may come from teaching and research institutions in France or abroad, or from public or private research centers.
L'archive ouverte pluridisciplinaire HAL, est destinée au dépôt et à la diffusion de documents scientifiques de niveau recherche, publiés ou non, émanant des établissements d'enseignement et de recherche français ou étrangers, des laboratoires publics ou privés. 


\title{
Photothermal microscopy: optical detection of small absorbers in scattering
}

\section{environments}

\author{
Pierre Vermeulen ${ }^{1,2}$, Laurent Cognet ${ }^{1,2}$, Brahim Lounis ${ }^{1,2^{*}}$
}

${ }^{1}$ Univ Bordeaux, LP2N, F-33405 Talence, France.

${ }^{2}$ Institut d'Optique \& CNRS, LP2N, F-33405 Talence, France.

Keywords: Photothermal imaging, microscopy, gold nanoparticles, single particle tracking.

\section{Summary}

Photothermal microscopy enables detection of nanometer-sized objects solely based on their absorption. This technique allows efficient observation of various nano-objects in scattering media notably gold nanoparticles in cells. The extreme sensitivity of the method and the stability of the signals open numerous applications in spectroscopy, analytical chemistry and bioimaging. This review briefly describes the principle and the main characteristics of photothermal microscopy, with its major advantages and limitations, and exposes the principal applications that have been carried out since its first implementation. 


\section{Introduction}

During the past decade, the development of sensitive microscopy techniques has provided numerous breakthroughs in nanoscience or biological studies. Currently, the majority of methods are based on fluorescence microscopy, as it allows high sensitivity detection of fluorescent objects, up to the single molecule level (Stender et al., 2013 a recent and extensive review). Moreover, the large toolbox of labelling strategies developed over the years enables the specific observation of virtually any cell constituents. However, the major drawback of these techniques is photobleaching, which limits the observation time on single fluorescent molecule to typically a few seconds. Other types of fluorescent nano-emitters, like quantum dots (Pinaud, Clarke, Sittner, \& Dahan, 2010), have been developed to face this problem but at the expense of bigger size (Groc et al., 2007) and lower biocompatibility.

In contrast to luminescence, absorption does not suffer from photobleaching and blinking, making it attractive for robust single molecule detection. Photothermal imaging (Phl) (Berciaud, Cognet, Blab, \& Lounis, 2004; Berciaud, Lasne, Blab, Cognet, \& Lounis, 2006; Boyer, Tamarat, Maali, Lounis, \& Orrit, 2002) is an indirect absorption detection technique which achieves high detection sensitivity while discriminating absorption from scattering. It is based on the detection of refractive index variations that are induced by photothermal effect in the local environment of an absorbing nanoparticle (Berciaud et al., 2004, 2006). This technique enables the detection of nano-objects with an absorption cross section as small as a few $\AA^{2}$ (Berciaud et al., 2004; Berciaud, Cognet, \& Lounis, 2005; Gaiduk, Yorulmaz, Ruijgrok, \& Orrit, 2010). Gold nanoparticles of a few nanometres are particularly suited for this imaging modality, thanks to their fast relaxation times (ps range) and large absorption sections around their plasmon resonance (for instance, $\sim 6 \mathrm{~nm}^{2}$ for $5 \mathrm{~nm}$ gold nanoparticles). They have been used in different applications in conjunction with photothermal detection modality (Berciaud, Cognet, \& Lounis, 2008; Berciaud, Cognet, Poulin, Weisman, \& Lounis, 2007; Berciaud et al., 2006; Berciaud, Cognet, \& Lounis, 2005; Berciaud, Cognet, Tamarat, \& Lounis, 2005; Blab et al., 
2006; Lasne et al., 2006; Octeau et al., 2009). In this review, we will describe the main features of the photothermal technique and present some of its applications.

\section{Detection principle and signal modelling}

Upon resonant $\mathrm{cw}$-laser excitation, small absorbers with minute luminescence yields convert all the absorbed energy into heat. This leads to a temperature rise of their local environment, which induces variations of the local refractive index. This photothermal effect can be efficiently probed using an additional non-resonant laser beam. In Phl microscopy, the two beams are superimposed and focussed in the sample using a microscope objective lens (Berciaud et al., 2004). The heating beam is modulated at high frequency $(\Omega / 2 \pi \sim 0.1-1 \mathrm{MHz}$ typically) and consequently the local temperature increase $\Delta T$ and the refractive index variations $\Delta n$ surrounding the particle are also modulated at the same frequency. The time-dependent refractive index profile induced around the absorber writes (Berciaud et al., 2006):

$$
\Delta n(r, t)=\frac{\partial n}{\partial T} \Delta T(r, t)=\frac{\partial n}{\partial T} \frac{\sigma_{a b s} \mathrm{I}_{\text {heat }}}{4 \pi \kappa r}\left[1+\exp \left(-\frac{\mathrm{r}}{\mathrm{r}_{\mathrm{th}}}\right) \cos \left(\Omega \mathrm{t}-\frac{\mathrm{r}}{\mathrm{r}_{\mathrm{th}}}\right)\right]
$$

where $r$ is the distance from the particle, $\frac{\partial n}{\partial T}$ the variations of the refractive index $n$ with temperature (typically $-10^{-4} \mathrm{~K}^{-1}$ ) and $\mathrm{r}_{\mathrm{th}}=\sqrt{\frac{2 \kappa}{\Omega \mathrm{C}}}$ the characteristic length for heat diffusion ( $\kappa$ being the thermal conductivity of the surrounding medium and $C$ its heat capacity). Moreover, $\sigma_{a b s} \mathrm{I}_{\text {heat }}$ is the power absorbed by a particle of absorption cross section $\sigma_{a b s}$, illuminated by a heating beam of intensity $I_{\text {heat }}$.

This refractive index profile is measured using a probe beam superimposed with the heating beam. The wavelength of the probe laser is chosen such that its absorption by the nano-object is minimal.

Several models have been developed to investigate the main parameters influencing $\mathrm{Phl}$ signals. The first model (Berciaud et al., 2006) provides an analytical expression of the photothermal signal derived using the theory of light scattering from a fluctuating medium. In this model the interaction 
of the incident probe field (approximated by a plane wave) with the local susceptibility fluctuations induced by the heated nanoparticle give rise to a local polarisation variations of the medium. The refractive index profile scatters this probe and the scattered field contains sidebands at the heating beam modulation frequency. Introducing the Hertz potential, which obeys to an inhomogeneous wave equation with the local polarization variations as a source term, the expression of the scattered field could be derived. The interferences of this scattered field with a reference field obtained from the probe beam are monitored with a photodiode and a lock-in demodulation at $\Omega$ extracts the signal revealing the presence of a nanoparticle. Depending on the chosen configuration (Berciaud et al., 2006) (cf. fig. 1a), the reference field is the transmitted probe field (forward configuration) or a reflection of the incident field, e.g. at the glass-sample interface (backward configuration). With this model the amplitudes of the signals detected in the backward and forward configurations can be compared and their frequency dependences can be studied (Berciaud et al., 2006):

$$
P_{P h I}=\eta \sqrt{\alpha} \mathrm{P}_{\text {probe }}\left(2 \pi \sqrt{2} \frac{\partial n}{\partial T} \frac{1}{c \lambda^{2} w_{0}} \mathrm{P}_{\text {abs }}\right) f(\Omega)
$$

where $\eta$ is the optical system transmission efficiency, $\alpha$ depends on the chose configuration and describes the amplitude of the reference field $(\alpha=R$, the power reflexion coefficient in the backward geometry, $\alpha=T$, the power transmission coefficient in the forward geometry), $w_{0}$ is the probe beam waist, $\lambda$ its wavelength and $\mathrm{P}_{\text {probe }}$ its power. $f(\Omega)$ is a function describing the complex frequency dependence of the signal, especially related to the diffusion length $r_{\text {th }}$ compared to the beam diameters. At frequencies higher than $500 \mathrm{kHz}$, the function $f(\Omega)$ and the photothermal signal decrease as $1 / \Omega$.

If the probe beam intensity is high enough, the detection is shot noise limited, and the resulting signal to noise ratio $S N R_{P h I}$ can be expressed as:

$$
S N R_{P h I} \propto \sqrt{\Delta t} \sqrt{\mathrm{P}_{\text {probe }}} \mathrm{P}_{\text {abs }}\left(\frac{\partial n}{\partial T} \frac{1}{C}\right) f(\Omega)
$$


with $\Delta t$ the integration time of the lock-in amplifier.

Following this formula, it is possible to optimize the photothermal signal noise ratio, by playing either on laser intensities, integration time, modulation frequency or also on the medium thermal properties (Gaiduk, Ruijgrok, Yorulmaz, \& Orrit, 2010; Octeau et al., 2009). Photodamage of the absorbing nanoparticle and excessive heat of the local medium (especially in biological environment) however set a limit to the intensity of the heating laser. Water is an embedding medium with good thermal properties allowing various biological applications of the photothermal technique (Lasne et al., 2006; Leduc et al., 2013; Leduc, Jung, Carney, Stellacci, \& Lounis, 2011; Rossier et al., 2012; Sée et al., 2009). Organic solvents or certain oils (e.g. glycerol, silicon) display optimized thermal properties that allow pushing the detection limits towards absorbers with very small absorption cross sections. Interestingly media exhibiting a phase transition around the working temperature can further increase the SNR because of large variations of refractive index around their critical temperature (Chang \& Link, 2012; Parra-Vasquez, Oudjedi, Cognet, \& Lounis, 2012).

In the second model, Cichos and co-workers (Selmke, Braun, \& Cichos, 2012a, 2012b) showed that using a full ab-initio calculation of a generalized Mie description, the long range refractive index change generated by a single heated nano-object in photothermal microscopy acts as a nano-lens. From the refractive index profile, the transmitted probe field is computed at steady state taking into account the use of highly focussed beams (heat and probe) instead of plane waves, the relative axial positions of their waists and aberration effects in the focussed laser beams. The photothermal signal is computed as the difference of the probe transmitted power between two steady states, in the presence and absence of the heating beam. This model sheds light on some important parameters as the dependence of the $\mathrm{Phl}$ signal with relative beam waist positions. In particular, the signal phase can change sign along the optical axis depending on the relative axial positions of the beams waists. This feature can be useful to extract 3D localisation and velocimetry (Selmke, Schachoff, Braun, \& Cichos, 2013). Besides, the maximum photothermal signal can be axially shifted relative to the 
maximum of excitation beams (Gaiduk, Ruijgrok, Yorulmaz, \& Orrit, 2011; Selmke et al., 2012a), which can limit the correlation of fluorescence and photothermal images. Finally, this model does not apply to the backward configuration and it is based on steady state calculations, with no investigation of the modulation frequency dependence of the signals.

An interesting analogy has recently been highlighted between photothermal imaging of a single particle and Rutherford scattering, as light is deflected by the presence of the index gradient as $\alpha$ particles on atoms (Selmke \& Cichos, 2013).

\section{Experimental setup}

Figure 1 presents the general elements of a photothermal microscope. A non-resonant probe beam (632.8 nm, HeNe, or single frequency Ti:Sa laser) and a modulated heating beam (single line laser or e.g. frequency doubled Nd:YAG laser or tunable $\mathrm{cw}$ laser for absorption spectroscopy) are overlaid and focussed on the sample using a high numerical aperture (NA) microscope objective (e.g. 100x, $N A=1.4)$. The intensity of the heating beam is modulated at $\Omega$ ranging from typ. $100 \mathrm{kHz}$ to the $\mathrm{MHz}$, by an acousto-optic modulator. The Phl signal can be detected using two different configurations. In the backward configuration, a combination of a polarizing cube and a quarter wave plate is used to extract the interfering probe-reflected and backward-scattered fields. In order to detect the forward signal, a second microscope objective (air or water immersion objective, with an optimal NA around 0.8 (Selmke et al., 2012a)) is employed to efficiently collect the interfering probe-transmitted and forward-scattered fields. The intensity of the heating beam illuminating the nanoparticles ranges from less than $1 \mathrm{~kW} / \mathrm{cm} 2$ to $\sim 5 \mathrm{MW} / \mathrm{cm} 2$ (depending on the desired signal-to-noise ratio, the nanoparticle absorption cross section and the maximum temperature rise admissible in the sample). Backward or forward interfering fields are collected on fast photodiodes and fed into a lock-in amplifier in order to extract the beat signal at $\Omega$. Integration time of 1 to $10 \mathrm{~ms}$ are typically used. Images are obtained by moving the sample over the fixed laser spots by means of a 2D piezo-scanner or by scanning the beam positions with respect to the sample with galvanometric mirrors. 


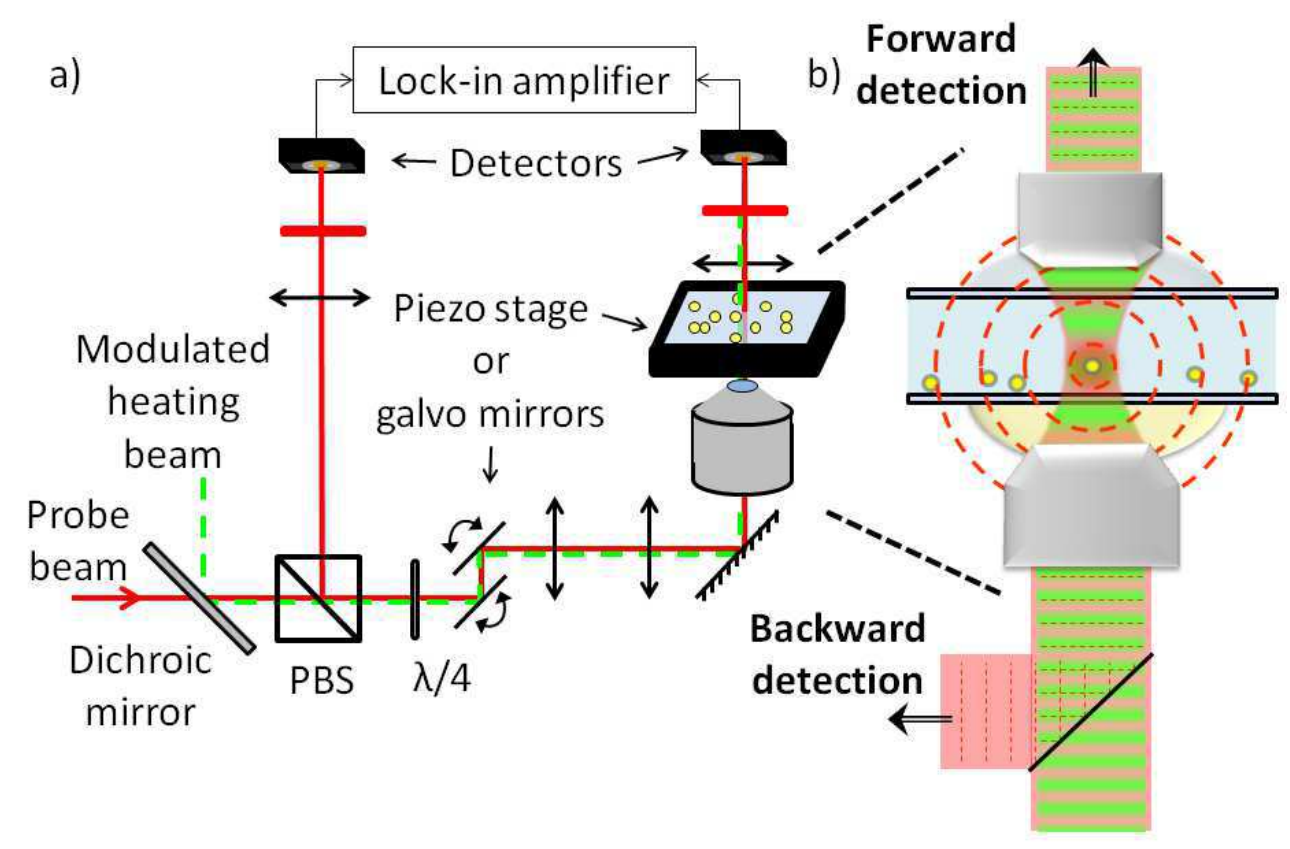

Figure 1: a) Typical photothermal setup (PBS: polarizing beam splitter, $\lambda / 4$ : quarter wave plate) with $b)$ backward and forward configurations.

The heating beam wavelength is chosen according to the absorber of interest. For gold nanoparticles, $532 \mathrm{~nm}$ is often used, as it is close to the maximum of the plasmon resonance. To reach the ultimate shot noise limit for the detection, probe lasers with the lowest intensity noise and relatively high modulation frequencies ( $100 \mathrm{kHz}-1 \mathrm{MHz}$ ) should be used.

Figure $2 \mathrm{a}$ ) and b) present photothermal images of $10 \mathrm{~nm}$ gold nanoparticles embedded in silicon oil and observed in the forward and backward configurations (heating intensity of $600 \mathrm{~kW} / \mathrm{cm}^{2}$, probe intensity of $3 \mathrm{MW} / \mathrm{cm}^{2}, \Delta \mathrm{t}=5 \mathrm{~ms}, \Omega=700 \mathrm{kHz}$ ). Single particles are detected with high signal noise ratio. The point-spread function of this technique depends on the probe and heating beam profiles and on their overlap but also on the dielectric susceptibility profile created around the nanoparticles. For tightly focussed beams, a lateral resolution of $\sim 250 \mathrm{~nm}$ is commonly achieved.

In addition to their excellent absorbing properties, gold nanoparticles present other benefits for biological imaging. Their synthesis is robust and produces monodisperse solutions of nanometer size beads. The absorption cross section of gold nanoparticles scaling with the volume of the particle, the signal dispersion of single nanoparticles is thus three times the nanoparticle size dispersion (Figures 
$2 \mathrm{e}$ and $2 \mathrm{f}$ ). This property can be used to perform stoichiometry measurements of biomolecules labelled with gold nanoparticles, in cells (Cognet et al., 2003) or DNA microarrays (Blab et al., 2006).
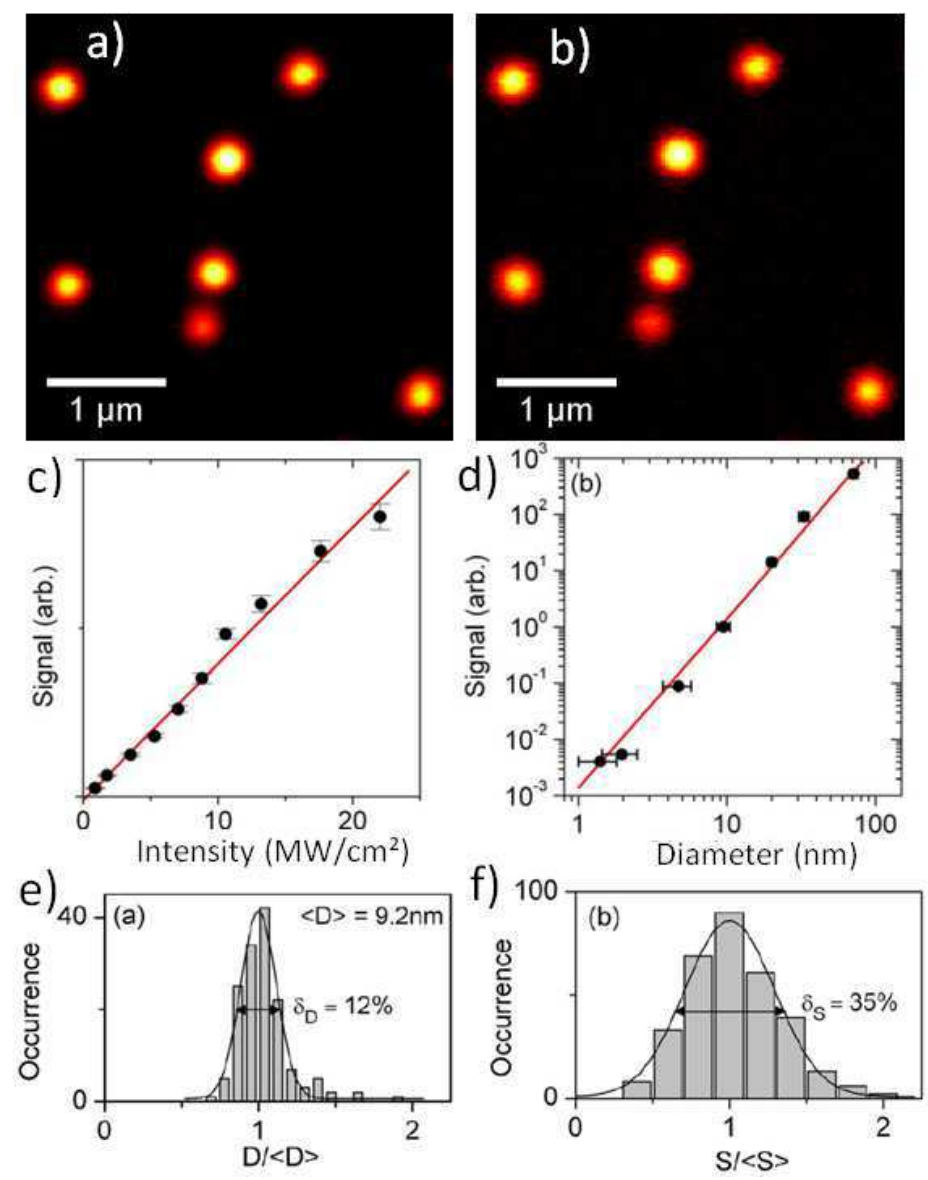

Figure 2: Phl images and dependences. a) and b) Images of $10 \mathrm{~nm}$ gold nanoparticles deposit on a glass slide, in the forward (SNR $=322)(a)$ and backward $(S N R=62)(b)$ configurations.

Photothermal signal dependence with c) heating power, measured from a single $10 \mathrm{~nm}$ nanoparticle and d) particle size. e) Diameter D (TEM measurements) and f) photothermal signal S distributions for $10 \mathrm{~nm}$ gold nanoparticles.

\section{Applications}

\section{Absorption spectroscopy}

Since photothermal signals are proportional to absorption, it can be used to perform absorption spectroscopy of individual nano-structures and therefore freed from inhomogeneous broadening of ensemble measurements. For example, it allowed studying the plasmon resonance of individual metallic nanoparticles smaller than $10 \mathrm{~nm}$ size (Berciaud, Cognet, Tamarat, et al., 2005) and measuring intrinsic broadening of the plasmon resonance due to enhanced dephasing by electron 
surface scattering. Absorption spectra of individual semiconductor nanocrystals in multi-exciton regime could also be recorded and biexciton and trion binding energies could be extracted (Berciaud, Cognet, \& Lounis, 2005). The application of this method to the absorption spectroscopy of single wall carbon nanotubes (both metal or semiconducting) was also performed at the single tube level (Berciaud et al., 2007) providing access to the absorption cross section of these objects (Berciaud et al., 2008).

\section{Photothermal absorption correlation spectroscopy}

Fluorescence correlation spectroscopy (FCS) is a popular technique for the investigation of dynamic processes of molecules. Based on fluorescence, this single molecule method suffers from artifacts originating from the poor fluorophore photophysics: photobleaching, blinking and saturation. To circumvent these limitations, photothermal detection of absorbing object has been developed as an alternative to fluorescence. Photothermal Absorption Correlation Spectroscopy (PhACS) is then the equivalent of FCS where the time autocorrelation of the photothermal signal is analyzed.

It allows quantitative determinations of the diffusion constant of absorbing nanoparticles and can be used to measure the hydrodynamic diameter of functionalized nanoparticles complexes with nanometer precisions (Octeau et al., 2009; Paulo et al., 2009; Radünz, Rings, Kroy, \& Cichos, 2009).

PhACS is not sensitive to any scattering background and finds numerous applications in live cell studies by giving access to long time scales that are not accessible for standard FCS experiments. As an example, PhACS has been applied to measure the diffusion coefficient of gold particles in cells cytosol, and estimate its viscosity (Leduc et al., 2011).

Recently, a twin-focus photothermal cross correlation spectroscopy has been developed (Selmke, Schachoff, et al., 2013). The method is based on the photothermal nano-lensing mechanism which provides two axially and sharply separated detection-subvolumes with opposite photothermal signals, creating the twin-focus. Correlation between positive and negative signals allows the 
measurement of slow axial dynamics $(10 \mathrm{~nm} / \mathrm{ms})$, which could be of interest to detect directed motion of gold labelled biomolecules in cells for example. In addition, study of Phl signal histogram can reveal properties of the diffusing particles such as concentration, size distribution, etc., from the same dataset (Selmke, Braun, Schachoff, \& Cichos, 2013).

\section{Applications to biological imaging: Single NanoParticle Tracking in Live cells}

The stability of photothermal signal is appealing for biological applications where photobleaching and blinking of fluorescent probes constitute a major limitation. Photothermal microscopy can be used for dense labelling and cells substructures imaging, as well as sparse labelling for single particle tracking even in confined and crowded environments such as synapses (Lasne et al., 2006) and adhesion sites (Leduc et al., 2013). Furthermore Phl is totally insensitive to non-absorbing scatterers, even when large objects with strong refractive index contrasts are present within the surroundings of the imaged nanoparticles, as is often the case in biological samples (Lasne et al., 2006).

Molecular imaging down to the single molecule level in such complex environments requires optimized probes. Indeed, an ideal probe should be very specific, as small as possible to minimize steric hindrance, monovalent, optically stable for long observation times and able to deliver intense optical signals for precise localization. To fully benefit from the small size of gold nanoparticles detectable by photothermal microscopy, the ligand used to bind the particle to the target biomolecule should then be small. Commonly used antibodies have typical sizes of $12 \mathrm{~nm}$ and thus significantly increase the overall probe size. A small and versatile absorbing probe dedicated to photothermal imaging of cell has recently been developed (Leduc et al., 2013). This probe, depicted on figure $3 \mathrm{a}$, is composed of a gold nanoparticle of $5 \mathrm{~nm}$ diameter functionalized with a GFPnanobody, a small fragment of antibody with a single binding domain ( $2 \mathrm{~nm} \times 4 \mathrm{~nm}$ ) with a high affinity for GFP. This construct gathers several crucial properties of an ideal probe. Its final size is less than $10 \mathrm{~nm}$ in diameter, allowing imaging in confined environments. Using an anti-GFP nanobody 
ensures specific labelling and versatility, thanks to the vast library of GFP-fused proteins. This probe is optically stable and generates bright signal, useful for long term tracking for example.
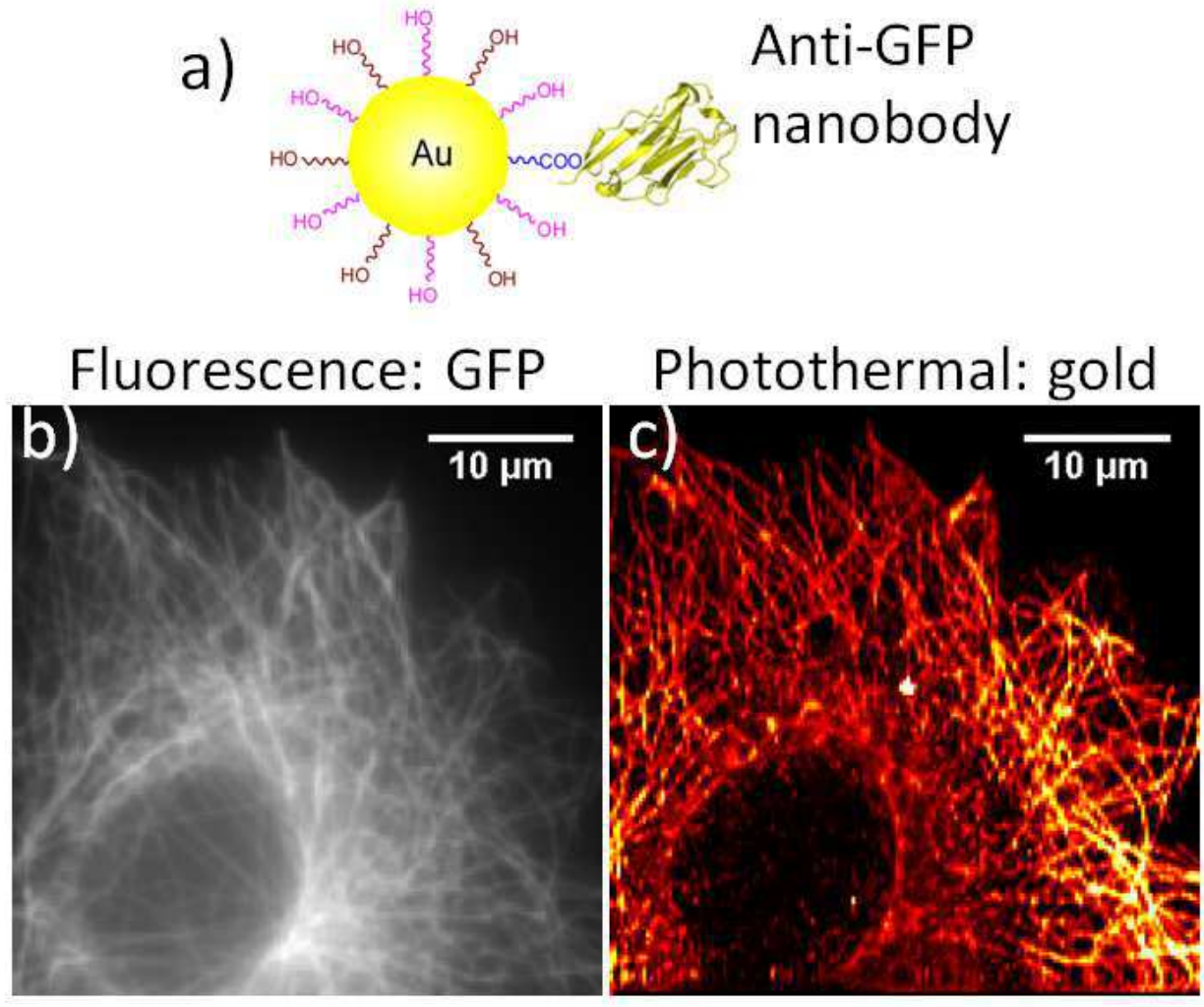

Figure 3: Bioimaging with photothermal contrast: a) probe design. Fluorescence b) and photothermal c) images of the same fixed COS-7 cell expressing EB3-GFP incubated with the nanoprobe, showing perfect colocalization and low non-specific signal.

Using this probe, surface and intracellular GFP-proteins were effectively labelled even in very crowded environments such as adhesion sites and cytoskeletal structures both in vitro and in live cell cultures (Leduc et al., 2013).

An important point for biological imaging is to control that the temperature increase due to the nanoparticles heating is kept low to ensure live cell integrity. In usual photothermal imaging conditions, the temperature elevation at the surface of the nanoparticles does not exceed a few degrees thanks to a limited heating beam power (Berciaud et al., 2006; Gaiduk, Ruijgrok, et al., 2010). 
The movements of molecules in the plasma membrane of living cells are characterized by their diversities, both in the temporal and spatial domain. Membrane proteins explore the plasma membrane in various lateral diffusion modes, and frequently interact with each other at specific locations. Robust and long-term tracking of single particles is required to investigate those mechanisms in detail. The photothermal technique is an attractive tracking technique, as it combines the advantages of single particle tracking which commonly uses large nanoparticles ( $>40 \mathrm{~nm}$ ) for efficient detection by Rayleigh scattering for very long times (but at the price of steric hindrance in confined environments) with those of single molecule fluorescence microscopy which uses very small probes (but at the price of photobleaching which severely limits the observation times of a single fluorophore to typically less than one second in live cells). Indeed, Phl microscopy allows long observation times of small nanometer-sized labels owing to the high chemical stability of gold particles. A photothermal image of live neurons with 5-nm-gold-labelled glutamate receptors on their outer membrane is presented in Figure 4a (compared to a white light image of the same area). As the Phl method requires a raster scan of the sample with typically a few-millisecond integration time per point, fast imaging rates cannot be readily obtained and moving objects are not resolved during the raster scan (Fig. 4a). Consequently, mobile receptors produce stripes of signal in the image. Lasne et al. designed a tracking scheme based on a triangulation from three measurement points (as used in the GPS scheme) to record the trajectories of single membrane proteins labelled with gold nanoparticles in live cells at video rate (fig $4 \mathrm{~b}$ ). The movement of single membrane receptors could thus be recorded for several minutes in different cellular systems (Lasne et al., 2006; Leduc et al., 2013).

Thanks to the high photothermal signal stability, recording the full history of proteins in cells including intermediate states even in highly confined regions is now possible, allowing the investigation of many biological questions, which are unreachable with other techniques. 

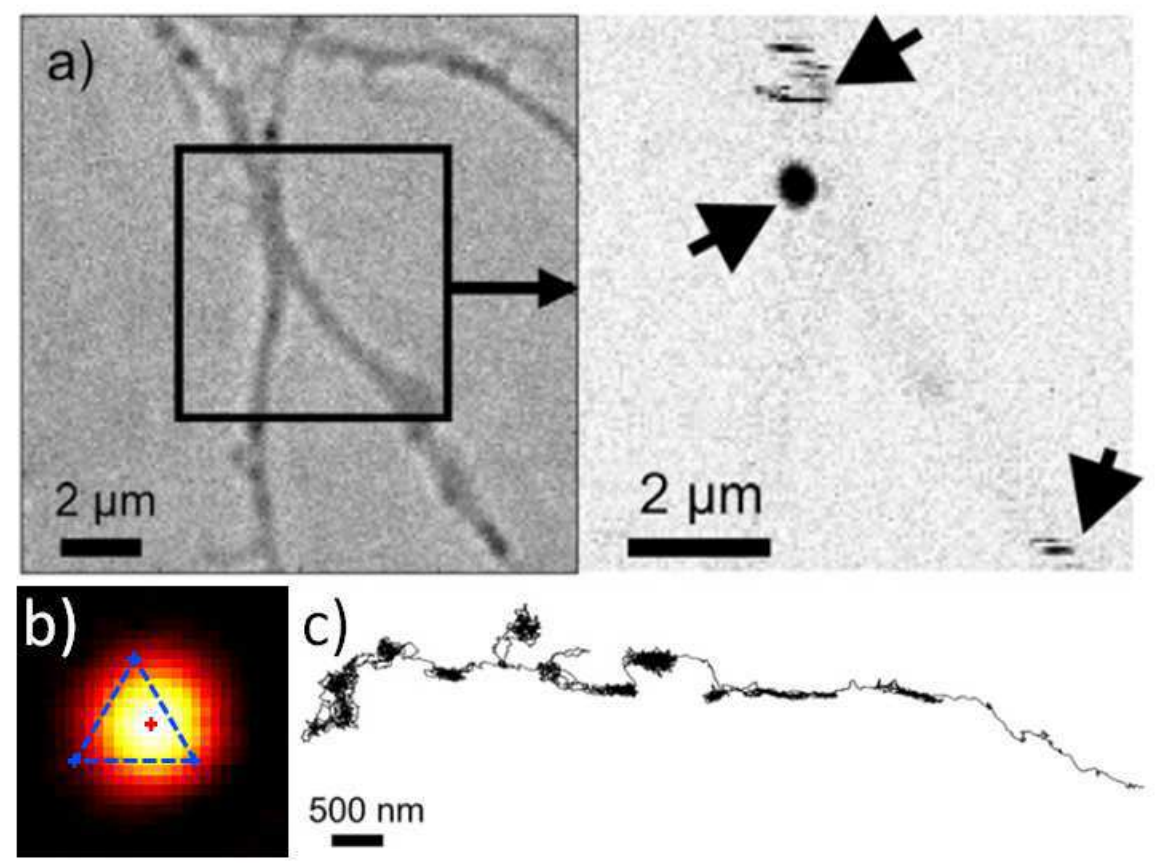

$500 \mathrm{~nm}$

Figure 4: White light image (a, left) and photothermal image (a, right) of a live neuron labelled with gold nanoparticles. Arrows indicates the presence of two moving (strips) and one stationary (spot) GluR2 receptors labelled with gold. b) Trajectory of an individual 5-nm gold nanoparticle (>9000 data points, $>5 \mathrm{~min}$ ), acquired at video rate on a live neuron (from (Lasne et al., 2006)). c) Localization procedure: three measurements taken at the vertices of an equilateral triangle (blue crosses) are sufficient to determine the position of the particle (red cross) and signal intensity.

\section{Discussion and conclusion}

Photothermal imaging is a powerful technique allowing optical detection of absorbing single nanoparticles on a dark background. Its sensitivity is large enough to detect particles with absorption cross section of a few $\AA^{2}$, such as single chromophore, $1.4 \mathrm{~nm}$ gold clusters, etc. The stability of the signal presents important advantages for many applications, especially in biological imaging, and thus offers interesting prospects.

There is however some limitations using photothermal microscopy for biological imaging. Unlike fluorescence, parallelisation using matrix detectors (CCD) is not possible because of the lock-in detection scheme. Photothermal microscopy thus remains a scanning technique (with typical 
integration times of the order of the millisecond per pixel) with limited applications in fast wide-field live cells imaging.

Another limitation arises from the intrinsic photothermal signal due to residual absorption of green light by cell mitochondria, which lead to a background signal. The use of gold nanorods as small probes absorbing in the near infrared, a region where the absorption of cell organelles is negligible, can solve this problem. One can also take advantage of this intrinsic absorption to achieve label-free imaging (Lasne et al., 2007).

Although more difficult to implement, photothermal multicolour imaging can be envisaged by multiplexing biomolecule labelling with metal nanoparticles having different tailored plasmon resonances, such as gold nanorods with different aspect ratios, or various core/shell particles.

Finally, photothermal microscopy being a far-field optical technique with an extreme sensitivity for gold nanoparticles, it reaches the same sensitivity as electron microscopy, but with standard optical resolution. Since cellular imaging can be performed with the same labelling procedures that the ones used for cryo-prepared sample in electron microscopy, the photothermal technique might represent a key technology to perform correlation microscopy between optical live cell imaging and electron microscopy ultrastructure.

\section{References}

Berciaud, S., Cognet, L., Blab, G., \& Lounis, B. (2004). Photothermal Heterodyne Imaging of Individual Nonfluorescent Nanoclusters and Nanocrystals. Physical Review Letters, 93(25), 257402. doi:10.1103/PhysRevLett.93.257402

Berciaud, S., Cognet, L., \& Lounis, B. (2005). Photothermal absorption spectroscopy of individual semiconductor nanocrystals. Nano letters, 5(11), 2160-3. doi:10.1021/nl051805d

Berciaud, S., Cognet, L., \& Lounis, B. (2008). Luminescence Decay and the Absorption Cross Section of Individual Single-Walled Carbon Nanotubes. Physical Review Letters, 101(7), 077402. doi:10.1103/PhysRevLett.101.077402

Berciaud, S., Cognet, L., Poulin, P., Weisman, R. B., \& Lounis, B. (2007). Absorption spectroscopy of individual single-walled carbon nanotubes. Nano letters, 7(5), 1203-7. doi:10.1021/nl062933k 
Berciaud, S., Cognet, L., Tamarat, P., \& Lounis, B. (2005). Observation of intrinsic size effects in the optical response of individual gold nanoparticles. Nano letters, 5(3), 515-8. doi:10.1021/nl050062t

Berciaud, S., Lasne, D., Blab, G., Cognet, L., \& Lounis, B. (2006). Photothermal heterodyne imaging of individual metallic nanoparticles: Theory versus experiment. Physical Review B, 73(4), 045424. doi:10.1103/PhysRevB.73.045424

Blab, G. a, Cognet, L., Berciaud, S., Alexandre, I., Husar, D., Remacle, J., \& Lounis, B. (2006). Optical readout of gold nanoparticle-based DNA microarrays without silver enhancement. Biophysical journal, 90(1), L13-5. doi:10.1529/biophysj.105.076182

Boyer, D., Tamarat, P., Maali, A., Lounis, B., \& Orrit, M. (2002). Photothermal imaging of nanometersized metal particles among scatterers. Science (New York, N.Y.), 297(5584), 1160-3. doi:10.1126/science.1073765

Chang, W.-S., \& Link, S. (2012). Enhancing the Sensitivity of Single-Particle Photothermal Imaging with Thermotropic Liquid Crystals. The Journal of Physical Chemistry Letters, 3(10), 1393-1399. doi:10.1021/jz300342p

Cognet, L., Tardin, C., Boyer, D., Choquet, D., Tamarat, P., \& Lounis, B. (2003). Single metallic nanoparticle imaging for protein detection in cells. Proceedings of the National Academy of Sciences of the United States of America, 100(20), 11350-5. doi:10.1073/pnas.1534635100

Gaiduk, A., Ruijgrok, P. V, Yorulmaz, M., \& Orrit, M. (2011). Making gold nanoparticles fluorescent for simultaneous absorption and fluorescence detection on the single particle level. Physical chemistry chemical physics]: PCCP, 13(1), 149-53. doi:10.1039/c0cp01389g

Gaiduk, A., Ruijgrok, P. V., Yorulmaz, M., \& Orrit, M. (2010). Detection limits in photothermal microscopy. Chemical Science, 1(3), 343. doi:10.1039/c0sc00210k

Gaiduk, A., Yorulmaz, M., Ruijgrok, P. V, \& Orrit, M. (2010). Room-temperature detection of a single molecule's absorption by photothermal contrast. Science (New York, N.Y.), 330(6002), 353-6. doi:10.1126/science.1195475

Groc, L., Lafourcade, M., Heine, M., Renner, M., Racine, V., Sibarita, J.-B., ... Cognet, L. (2007). Surface trafficking of neurotransmitter receptor: comparison between single-molecule/quantum dot strategies. The Journal of neuroscience?: the official journal of the Society for Neuroscience, 27(46), 12433-7. doi:10.1523/JNEUROSCI.3349-07.2007

Lasne, D., Blab, G. a, De Giorgi, F., Ichas, F., Lounis, B., \& Cognet, L. (2007). Label-free optical imaging of mitochondria in live cells. Optics express, 15(21), 14184-93. Retrieved from http://www.ncbi.nlm.nih.gov/pubmed/19550692

Lasne, D., Blab, G. a., Berciaud, S., Heine, M., Groc, L., Choquet, D., ... Lounis, B. (2006). Single Nanoparticle Photothermal Tracking (SNaPT) of 5-nm Gold Beads in Live Cells. Biophysical Journal, 91(12), 4598-4604. doi:10.1529/biophysj.106.089771

Leduc, C., Jung, J., Carney, R. R., Stellacci, F., \& Lounis, B. (2011). Direct Investigation of Intracellular Presence of Gold Nanoparticles via Photothermal Heterodyne Imaging. ACS nano, 5(4), 25872592. 
Leduc, C., Si, S., Gautier, J., Soto-Ribeiro, M., Wehrle-Haller, B., Gautreau, A., ... Lounis, B. (2013). A highly specific gold nanoprobe for live-cell single-molecule imaging. Nano letters, 13(4), 148994. doi:10.1021/nl304561g

Octeau, V., Cognet, L., Duchesne, L., Lasne, D., Schaeffer, N., Fernig, D. G., \& Lounis, B. (2009). Photothermal Absorption Correlation Spectroscopy. ACS nano, 3(2), 345-350.

Parra-Vasquez, a. N. G., Oudjedi, L., Cognet, L., \& Lounis, B. (2012). Nanoscale Thermotropic Phase Transitions Enhancing Photothermal Microscopy Signals. The Journal of Physical Chemistry Letters, 3(10), 1400-1403. doi:10.1021/jz300369d

Paulo, P. M. R., Gaiduk, A., Kulzer, F., Krens, S. F. G., Spaink, H. P., Schmidt, T., \& Orrit, M. (2009). Photothermal Correlation Spectroscopy of Gold Nanoparticles in Solution †. The Journal of Physical Chemistry C, 113(27), 11451-11457. doi:10.1021/jp806875s

Pinaud, F., Clarke, S., Sittner, A., \& Dahan, M. (2010). Probing cellular events, one quantum dot at a time. Nature methods, 7(4), 275-85. doi:10.1038/nmeth.1444

Radünz, R., Rings, D., Kroy, K., \& Cichos, F. (2009). Hot brownian particles and photothermal correlation spectroscopy. The journal of physical chemistry. A, 113(9), 1674-7. doi:10.1021/jp810466y

Rossier, O., Octeau, V., Sibarita, J.-B., Leduc, C., Tessier, B., Nair, D., ... Giannone, G. (2012). Integrins $\beta 1$ and $\beta 3$ exhibit distinct dynamic nanoscale organizations inside focal adhesions. Nature cell biology, 14(10), 1057-67. doi:10.1038/ncb2588

Sée, V., Free, P., Cesbron, Y., Nativo, P., Shaheen, U., Rigden, D. J., ... Lévy, R. (2009). Cathepsin L Digestion of Nanobioconjugates upon Endocytosis. ACSNano, 3(9), 2461-2468.

Selmke, M., Braun, M., \& Cichos, F. (2012a). Photothermal Single-Particle Microscopy: Detection of a Nanolens. ACS Nano, 6(3), 2741-2749. doi:10.1021/nn300181h

Selmke, M., Braun, M., \& Cichos, F. (2012b). Nano-lens diffraction around a single heated nano particle. Optics express, 20(7), 8055-8070.

Selmke, M., Braun, M., Schachoff, R., \& Cichos, F. (2013). Photothermal signal distribution analysis (PhoSDA). Physical Chemistry Chemical Physics, 15(12), 4250. doi:10.1039/c3cp44092c

Selmke, M., \& Cichos, F. (2013). Photothermal Single Particle Rutherford Scattering Microscopy. Physical Review Letters, 110(10), 103901. doi:10.1103/PhysRevLett.110.103901

Selmke, M., Schachoff, R., Braun, M., \& Cichos, F. (2013). Twin-focus photothermal correlation spectroscopy. RSC Advances, 3(2), 394. doi:10.1039/c2ra22061j

Stender, A. S., Marchuk, K., Liu, C., Sander, S., Meyer, M. W., Smith, E. a, ... Fang, N. (2013). Single cell optical imaging and spectroscopy. Chemical reviews, 113(4), 2469-527. doi:10.1021/cr300336e 